\title{
AÇĀO PREDADORA DO TUCUNARÉ (CICHLA OCELLARIS) SOBRE A TILÁPIA DO CONGO (TILAPIA RENDALLI) (OSTEICHTHYES, CICHLIDAE)
}

HITOSHI NOMURA ${ }^{1}$ J. F. S. MENEZES ${ }^{2}$ M. B. F. A. SOUZA ${ }^{2}$

\section{ABSTRACT}

An extensive culvivation of the cichlids Tilapia rendalli and its predator Cichla ocellaris was undertaken at a lake in the Ribeirão Preto (State of São Paulo) Campus of the University of São Paulo. Comparison of the results with former ones, obtained before the introduction of the predator, showed a considerable increase of tilapias, both in total length and total weight, showing the advantage of rearing them together.

\section{INTRODUÇÃO}

Para se controlar a reprodução excessiva, que provoca superpovoamento no caso das tilápias, um dos métodos de criação desses ciclídeos se baseia na utilização de um predador (Huet \& Timmermans, 1974). Morais Filho (1972) chegou a analisar o "black-bass" (Micropterus salmoides) e o tucunaré (Cichla ocellaris), na proporção de um predador para dezesseis tilápias, no período de engorda.

Nomura \& Seixas (1970) estudaram posteriormente a tilápia do Congo (Tilapia rendalli), criada extensivamente no lago do Campus da Universidade de São Paulo em Ribeirão Preto, Estado de São Paulo. Nessa época, só ocorria essa espécie no lago, introduzida que fora em 1957. Para dar combate ao excesso de tilápias, foram colocados por esses autores, em 1973, cinco tucunarés (Cichla ocellaris) adultos. Em pouco tempo essa espécie carnívora amazonense se adaptou ao novo ambiente e começou a se reproduzir.

Visando mostrar a ação predadora do tucunaré apresentamos a relação idade-comprimento total e comprimento total-peso total, comparando os dados do presente trabalho com o trabalho de Nomura \& Seixas (1970).

\section{MATERIAL E MÉTODOS}

O material constou de 50 exemplares, sendo 36 machos e 14 fêmeas, capturados com tarrafa. Infelizmente, devido ao estouro da comporta do lago, este se esvaziou completamente no dia 23 de outubro de 1980 e, por isso, a amostragem não foi maior, ocasionando a lacuna de dados, principalmente de fêmeas.

No laboratório os peixes foram medidos em milímetros e pesados em gramas, tendo-se retirado algumas escamas detrás da nadadeira peitoral esquerda de cada exemplar; depois foram dissecados e identificados os sexos. Após cozimento e retirada da carne, foi feita a contagem do número de vértebras; estas e as escamas foram utilizadas para determinação da idade dos peixes, tendo sido analisadas com um microscópio estereoscópico Wild.

Os peixes cujas vértebras e/ou escamas não apresentavam anéis ou tinham um na margem foram considerados como pertencentes à classe I de idade, e assim por diante. O comprimento médio quando da formação dos anéis II e III das fêmeas foi determinado por retro-cálculo, uma vez que exemplares com essas idades não apareceram na amostra. No retro-cálculo

Trabalho realizado com auxílio da Fundaçăo de Amparo à Pesquisa do Fistado de São Paulo.

1. Departamento de Zootecnia, Escola Superior de Agricultura Luiz de Queiroz, Universidade de Sâo Paulo.

2. Departamento de Biologia, Faculdade de Filosofia, Ciências e Letras de Ribeirão Preto, Universidade de São Paulo. 
foram tomadas as medidas do foco das escamas e/ou vértebras até os anéis II e III, assim como até a margem. Considerando-se o crescimento dessas estruturas como proporcional ao crescimento do peixe, usamos uma regra de três: a distância do foco da estrutura até a margem corresponde ao comprimento total do peixe, enquanto que a do foco até os anéis II e III corresponde ao comprimento total nessas idades. Foi também calculada a porcentagem do aumento em comprimento total, por idade, assim como em peso total.

Os comprimentos totais foram agrupados em classes de $1 \mathrm{~cm}$ e a média dos pesos correspondentes foi extraída. Para relacionar o comprimento total com o peso total aplicamos a fórmula:

$$
\mathrm{W}=\mathrm{a} \mathrm{L}^{\mathrm{b}} \text { (Ricker, 1958) }
$$

onde: $\mathrm{W}=$ peso em gramas; $\mathrm{L}=$ comprimento total em milímetros; $\mathrm{a}, \mathrm{b}$, $=$ constantes, calculadas através da reta de regressão dos valores logarítmicos:

$$
\log \mathrm{W}=\log \mathrm{a}+\mathrm{b} \log \mathrm{L}
$$

pelo método dos mínimos quadrados.

\section{RESULTADOS}

Idade-comprimento total - A Tabela I e Figura 1 mostram os resultados obtidos. Os dados foram comparados com aqueles apresentados por Nomura \& Seixas (1970).

Foi insignificante a diferença de idades (comprimento médio) obtidas pelas análises das escamas e das vértebras, podendo-se usar uma ou outra estrutura.

A Tabela II mostra as diferenças em comprimento total, por idade, também expressas em aumento relativo, entre os dados anteriores (Nomura \& Seixas, 1970) e os do presente trabalho (análise das escamas). Na idade I o aumento relativo em comprimento foi de $38,82 \%$ nos machos e $42,35 \%$ nas fêmeas, tendo sido menores nas outras idades.

Comprimento total-peso total - A Tabela III e Figura 2 mostram os dados sobre esses dois parâmetros. Comparados com os do trabalho de Nomura \& Seixas (1970) nota-se que o maior aumento relativo do peso $(47,23 \%)$ foi com o da classe de $14 \mathrm{~cm}$ de comprimento total, tendo sido menor nas outras classes dos machos, enquanto que nas fêmeas só foi possível a comparação entre duas classes.

As equações que relacionam os dois parâmetros são:

$$
\begin{array}{ll}
\text { machos: } & \log \mathrm{W}=-1,735+3,044 \log \mathrm{X} \\
\text { fêmeas: } & \log \mathrm{W}=-1,497+2,827 \log \mathrm{X}
\end{array}
$$

Tabela I - Idade da tilápia-do-Congo, Tilapia rendalli, determinada pela leitura de escamas e vértebras, comparada com os dados de Nomura \& Seixas (1970) - comprimento em $\mathrm{cm}$

Nomura \& Seixas

Escamas:

Idade

(anos)

III

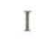

II

IV

V

machos

8,5

11,2

13,4

17,3

...

\section{fêmeas}

10,2

11,8

16,9

16,9

...

Escamas:

Nomura et al.
Vértebras machos fêmeas

$\begin{array}{cccc}\text { machos } & \text { fêmeas } & \text { machos } & \text { fêmeas } \\ 11,8 & 12,1 & 12,3 & 11,4 \\ 12,8 & 12,8^{*} & 13,0 & 12,6^{*} \\ 15,3 & 15,0^{*} & 15,2 & 14,8^{*} \\ 21,5 & 20,8 & 21,0 & 20,5 \\ 27,7 & 22,0 & 27,7 & 21,9\end{array}$




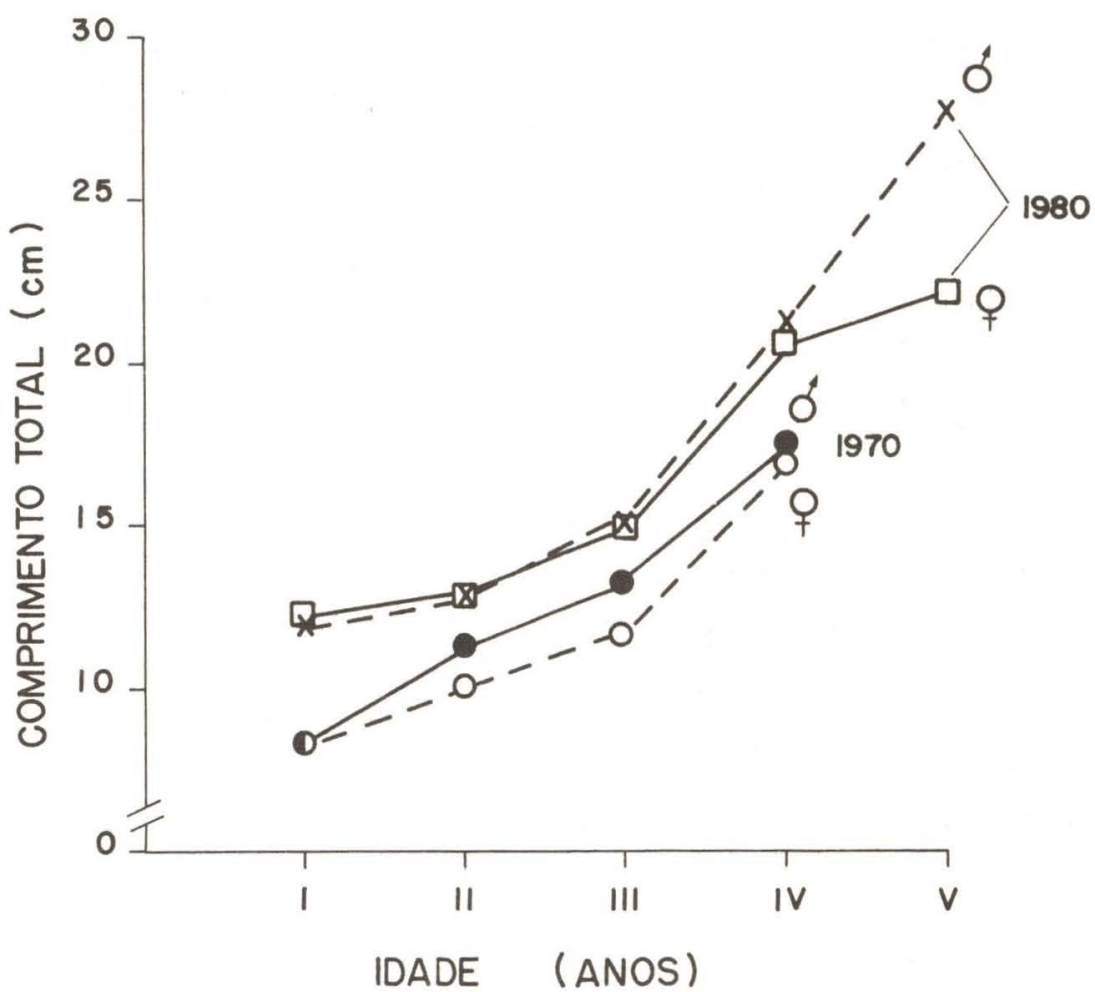

Figura 1 - Relação idade (anos) - comprimento total $(\mathrm{cm})$ da tilápia-doCongo, Tilapia rendalli, determinada em 1970 e 1980; neste último caso, após consorciação com o tucunaré, Cichla ocellaris.

Tabela II - Aumento absoluto (comprimento em $\mathrm{cm}$ ) e relativo (\%) das diferenças observadas por idades, determinadas pela leitura de escamas entre 1970 e 1980

1980

machos

fêmeas

$\begin{array}{rcccc}\begin{array}{r}\text { Idade } \\ \text { (anos) }\end{array} & \begin{array}{c}\text { aumento } \\ \text { absoluto }\end{array} & \begin{array}{c}\text { aumento } \\ \text { relativo }\end{array} & \begin{array}{c}\text { aumento } \\ \text { absoluto }\end{array} & \begin{array}{c}\text { aumento } \\ \text { relativo }\end{array} \\ \text { I } & 3,3 & 38,82 & 3,6 & 42,35 \\ \text { II } & 1,6 & 14,28 & 2,6 & 25,49 \\ \text { III } & 1,9 & 14,17 & 3,2 & 27,11 \\ \text { IV } & 4,2 & 24,27 & 3,9 & 23,07\end{array}$




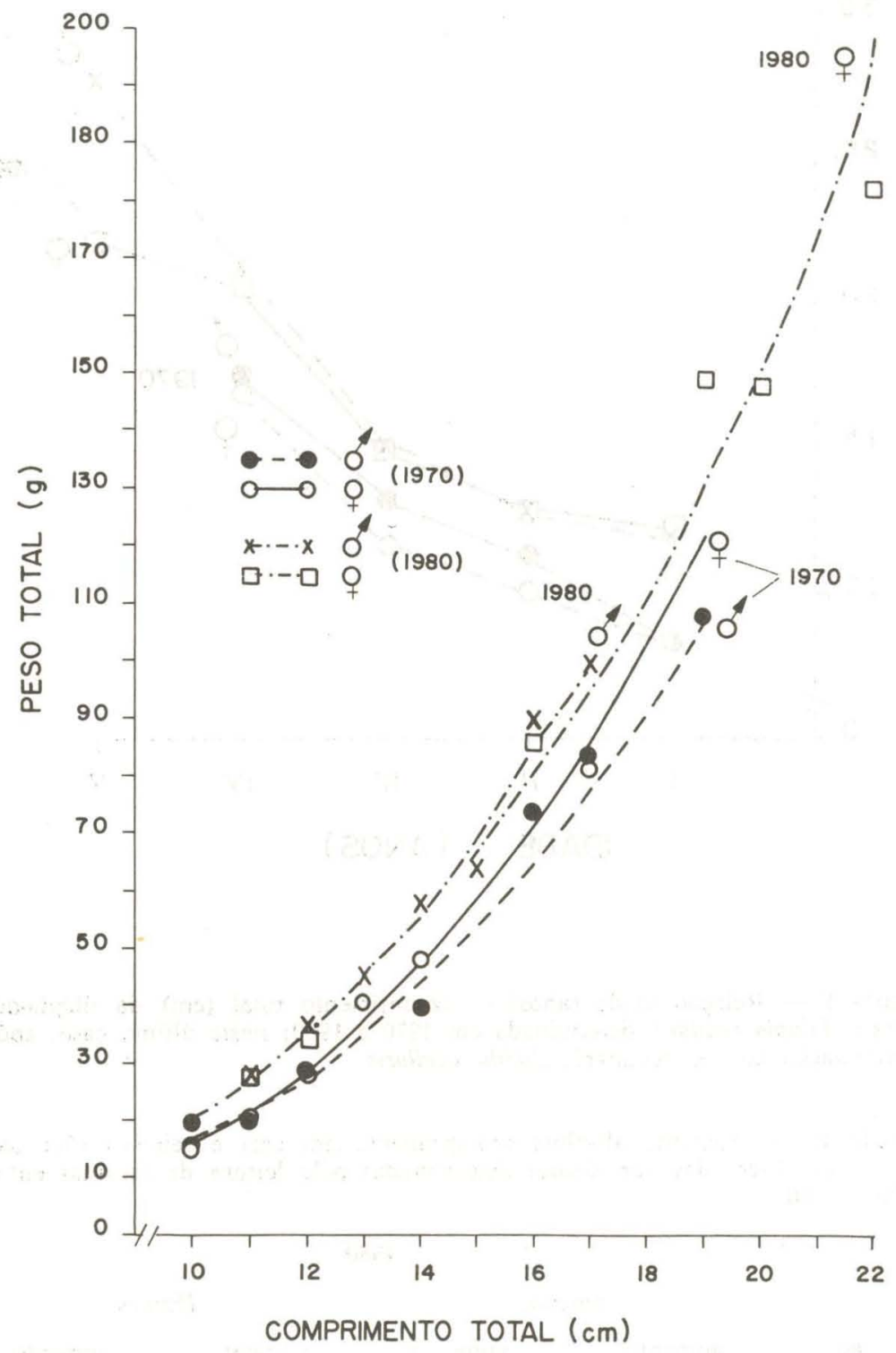

Figura 2 - Relação comprimento total $(\mathrm{cm})$ - peso total $(\mathrm{g})$ da tilápia-do-Congo, Tilapia rendalli determinada em 1970 e 1980; neste último caso, após consorciação com o tucunaré, Cichla ocellaris. 
Tabela III - Relação comprimento total (cm)-peso total (g) dos dados de 1970 e 1980, mostrando o aumento absoluto e relativo

\begin{tabular}{|c|c|c|c|c|c|c|c|c|}
\hline $\begin{array}{c}\text { Compr. } \\
\text { total }\end{array}$ & $\begin{array}{l}\text { peso } \\
1970\end{array}$ & $\begin{array}{c}\text { dos ma } \\
1980\end{array}$ & $\begin{array}{l}\text { 10s: at } \\
\text { absol }\end{array}$ & $\begin{array}{l}\text { hento } \\
\text { relat. }\end{array}$ & $\begin{array}{c}\text { pes } \\
1970\end{array}$ & $\begin{array}{l}\text { o das fé } \\
1980\end{array}$ & $\begin{array}{l}\text { neas: } \\
\text { absol. }\end{array}$ & $\begin{array}{l}\text { aumentc } \\
\text { relat. }\end{array}$ \\
\hline 10 & 20,1 & 19,7 & - & - & 14,6 & & - & - \\
\hline 11 & 20,4 & 28,0 & 7,6 & 37,25 & 21,2 & 27,8 & 6,6 & 31,13 \\
\hline 12 & 28,7 & 36,6 & 7,9 & 27,52 & 28,2 & 34,5 & 6,3 & 22,34 \\
\hline 13 & & 45,4 & - & - & 41,3 & $\ldots$ & - & - \\
\hline 14 & 39,8 & 58,6 & 18,8 & 47,23 & 48,4 & $\ldots$ & - & - \\
\hline 15 & & & - & - & $\ldots$ & $\ldots$ & - & - \\
\hline 16 & 73,6 & 90,1 & 16,5 & 22,41 & $\ldots$ & 86,5 & - & - \\
\hline 17 & 83,5 & 100,0 & 17,5 & 20,95 & 81,5 & $\ldots$ & - & - \\
\hline 18 & & $\ldots$ & - & - & $\ldots$ & $\ldots$ & - & - \\
\hline 19 & 108,5 & $\ldots$ & - & - & $\ldots$ & 148,6 & - & - \\
\hline 20 & $\ldots$ & $\ldots$ & - & - & $\ldots$ & 147,5 & - & - \\
\hline 21 & $\ldots$ & $\ldots$ & - & - & $\ldots$ & & - & - \\
\hline 22 & $\ldots$ & $\ldots$ & - & - & $\ldots$ & 176,9 & - & - \\
\hline
\end{tabular}

\section{DISCUSSAO}

Pelo exposto chega-se à conclusão de que é conveniente usar o tucunaré para predar a tilápia-do-Congo, a fim de evitar a superpopulação. Em nosso meio, apenas Morais Filho (1972) mostrou o resultado que conseguiu em 1958, no Posto de Piscicultura de Itapina, usando tanto o "black-bass" quanto - tucunaré, na proporção de 1 deles para 16 tilápias, tendo obtido 9680 $\mathrm{kg} / \mathrm{ha} / \mathrm{ano}$, com temperatura média da água de $26^{\circ} \mathrm{C}$ e $\mathrm{pH} 7$. O mesmo autor informa que Fuad Alzuguir utilizou 1 "black-bass" para 20 tilápias, em Pirassununga, SP, tendo obtido $8350 \mathrm{~kg} / \mathrm{ha} / \mathrm{ano}$, com temperatura que variou de 12 a $32^{\circ} \mathrm{C}$.

Galli (1981) recomenda a proporção de $15 \%$ de tucunarés para $85 \%$ de tilápias em locais quentes e $15 \%$ de "black-bass" em locais frios.

Visto os resultados alcançados no comprimento total e peso total, vale a pena criar tilápia do Congo em consorciação com o tucunaré.

\section{REFERENCIAS}

Galli, L. F., 1981. Introdução à piscicultura, ii +77 pp., 41 figs. Fundação Cargill, Campinas.

Huet, M. \& J. A. Timmermans, 1974. Textbook of fish culture, xii +436 pp., 503 figs. Fishing News (Books) Ltd., London.

Morais Filho, M. B., 1972. A tilápia, 37 pp., 5 figs. Superintendência de Desenvolvimento da Pesca, Rio de Janeiro.

Nomura, H. \& M. H. Seixas, 1970. Considerações sobre a biologia da tilápia, Tilapia melanopleura Duméril, 1859, da Fazenda Monte Alegre (Ribeirão Preto, São Paulo). Ciênc. Cult., S Paulo 22(3): 199-205, 5 figs.

Ricker, W. E., 1958, Handbook of computation for biological statistics of fish populations. Bull. Fish. Res, Bd Can. 119: 1-300, il. 